\title{
Effect of Cervical Sympathetic Trunk Transection on Renal Sympathetic Nerve Activity in Rats
}

\author{
T. IKEDA ${ }^{1}$, H. HIRAKAWA ${ }^{3}$, T. KEMURIYAMA ${ }^{4}$, Y. NISHIDA ${ }^{4}$, T. KAZAMA ${ }^{2}$ \\ ${ }^{1}$ Department of Anesthesiology, National Defense Medical College, Tokorozawa, and OUTCOMES \\ RESEARCH Consortium, ${ }^{2}$ Department of Anesthesiology, National Defense Medical College, \\ Tokorozawa, ${ }^{3}$ Department of Internal Medicine, Chikuhou Hospital, Fukuoka, ${ }^{4}$ Department of \\ Physiology, National Defense Medical College, Tokorozawa, Japan
}

Received December 27, 2006

Accepted September 17, 2007

On-line January 17, 2008

\begin{abstract}
Summary
Stellate ganglion blockade (SGB) with a local anesthetic increases muscle sympathetic nerve activity in the tibial nerve in humans. However, whether this sympathetic excitation in the tibial nerve is due to a sympathetic blockade in the neck itself, or due to infiltration of a local anesthetic to adjacent nerves including the vagus nerve remains unknown. To rule out one mechanism, we examined the effects of cervical sympathetic trunk transection on renal sympathetic nerve activity (RSNA) in anesthetized rats. Seven rats were anesthetized with intraperitoneal urethane. RSNA together with arterial blood pressure and heart rate were recorded for $15 \mathrm{~min}$ before and $30 \mathrm{~min}$ after left cervical sympathetic trunk transection. The baroreceptor unloading RSNA obtained by decreasing arterial blood pressure with administration of sodium nitroprusside was also measured. Left cervical sympathetic trunk transection did not have any significant effects on RSNA, baroreceptor unloading RSNA, arterial blood pressure, and heart rate. These data suggest that there was no compensatory increase in RSNA when cervical sympathetic trunk was transected and that the increase in sympathetic nerve activity in the tibial nerve during SGB in humans may result from infiltration of a local anesthetic to adjacent nerves rather than a sympathetic blockade in the neck itself.
\end{abstract}

\section{Key words}

Rat $\bullet$ Stellate ganglion

\section{Corresponding author}

Dr. Takehiko Ikeda, Department of Anesthesiology, National Defense Medical College, 3-2 Namiki, Tokorozawa 359-8513, Japan. Fax: +81-4-2992-1215. E-mail: tikeda@nyc.odn.ne.jp.

\section{Introduction}

Stellate ganglion blockade (SGB) is one of the common methods for the treatment of variety of sympathetically mediated diseases including postherpetic neuralgia (Milligan and Nash 1985) and reflex sympathetic dystrophy (Owen-Falkenberg and Olsen 1992). SGB induces Horner's syndrome (Malmqvist et al. 1992), and increases skin temperature (Stevens et al. 1998), and skin blood flow (Kim et al. 1975) in the cervicothoracic region and upper extremity because of suppression of sympathetic activity in these regions. Besides these effects, we have previously reported that left SGB with a local anesthetic mepivacaine increases muscle sympathetic nerve activity in the tibial nerve by direct intraneural recordings in humans (Ikeda et al. 1996). The precise mechanism for this increase in muscle sympathetic nerve activity in the tibial nerve during SGB remains unclear. One of the possible mechanisms is the blockade of vagal impulses from aortic baroreceptors by a local anesthetic. The vagal fibers from aortic baroreceptors run besides the sympathetic ganglion and it has been reported that solution injected percutaneously at the C6 or C7 level in humans spreads widely to surrounding areas (Guntamukkala and Hardy 1991, Honma et al. 2000). Another possible mechanism is compensatory arterial and/or cardiopulmonary baroreflex during vasodilation induced by sympathetic blockade in the neck. Whether the increase in muscle sympathetic nerve activity in the tibial nerve is due to infiltration of a local anesthetic to adjacent nerves or due to a cervical

PHYSIOLOGICAL RESEARCH • ISSN 0862-8408 (print) • ISSN 1802-9973 (online)

(c) 2009 Institute of Physiology v.v.i., Academy of Sciences of the Czech Republic, Prague, Czech Republic

Fax +420 241062 164, e-mail: physres@biomed.cas.cz, www.biomed.cas.cz/physiolres 
sympathetic blockade itself remains unknown. It is impossible to block selectively sympathetic ganglion in the neck per se using local anesthetics in humans. In addition, it was difficult to identify the vagal fibers from aortic baroreceptors in rats in our preliminary study. In the present study, we therefore investigated the effect of cervical sympathetic trunk transection on renal sympathetic nerve activity (RSNA) together with arterial blood pressure and heart rate in anesthetized rats. We measured RSNA, not tibial muscle sympathetic nerve activity in rats, since it is technically difficult to measure tibial muscle sympathetic nerve activity in rats. We also examined the maximum RSNA in response to a decrease in arterial blood pressure in order to evaluate baroreceptor unloading RSNA.

\section{Methods}

This study was performed in accordance with the guidelines of the Physiological Society of Japan and approved by the institutional Ethics Committee of Animal Care and Experimentation.

\section{Surgery}

Seven male Sprague-Dawley rats weighing between 400 and $500 \mathrm{~g}$ were anesthetized with intraperitoneal urethane $(1 \mathrm{~g} / \mathrm{kg})$. No additional anesthetics were administered throughout the study period. Ambient temperature was maintained near $25^{\circ} \mathrm{C}$. Rectal temperature was measured by a thermocouple and maintained between $36.5^{\circ} \mathrm{C}$ and $37.5^{\circ} \mathrm{C}$ using a radiant warmer. A heparin-filled polyethylene catheter was inserted into the abdominal aorta from left femoral artery to measure arterial blood pressure. Heparin-filled doublelumen polyethylene catheter was inserted into the inferior vena cava from the left femoral vein to administer phenylephrine or sodium nitroprusside for determining the background noise of RSNA, and the maximum RSNA, respectively. After a middle incision on the ventral region of the neck, the left common carotid artery, the left vagus nerve, the left superior laryngeal nerve, and the left cervical sympathetic trunk were identified. A surgical thread was passed around the cervical sympathetic trunk and both ends of the thread were put through a $22 \mathrm{G}$ needle for later transection. Through a left flank incision, a branch of the left renal nerve running on or beside the renal artery was exposed and dissected free from surrounding tissue under a dissecting microscope. Stainless steel bipolar hooked electrodes were used to record RSNA (Hirakawa et al. 1997). The measured RSNA might include afferent renal nerve activity to some extent. The nerve and electrodes were stabilized with a two-compartment silicone rubber gel. After the gel hardened, the incision was closed.

\section{Recording}

The arterial catheter was connected to a pressure transducer (DX-360; Nihon Kohden, Tokyo, Japan). The arterial blood pressure and heart rate were measured using a polygraph system (Nihon Kohden). RSNA electrodes were connected to amplifier systems. The original RSNA was amplified with a gain of 40000 and filtered $(50-1000 \mathrm{~Hz})$ by a differential low-noise amplifier (AVB-11, Nihon Kohden) and monitored continuously on an oscilloscope (VC-11, Nihon Kohden) and an audio speaker. Whole nerve activity was rectified, and the mean RSNA was obtained from the rectified signal by filtering at a time constant of $20 \mathrm{~ms}$. The pulsatile arterial blood pressure, mean arterial pressure, beat-to-beat heart rate, raw RSNA, and mean RSNA were recorded on an analog recorder (Nihon Kohden). The output from the analog recorder was connected to an analog-digital converter (PowerLab; AD Instruments, Castle Hill, Australia), and digitized values were displayed and recorded using a Macintosh personal computer.

\section{Experimental protocol}

Resting values of RSNA, arterial blood pressure, and heart rate before left cervical sympathetic trunk transection were recorded for $15 \mathrm{~min}$. Then, left cervical sympathetic trunk was transected by pulling the thread that was passed around the trunk in advance. RSNA, arterial blood pressure, and heart rate were recorded for 30 min after the transection. The maximum RSNA in response to the decrease in arterial blood pressure (the baroreceptor unloading RSNA) were measured twice; before $15 \mathrm{~min}$ of pre-transection period and after $30 \mathrm{~min}$ of post-transection period. To obtain the baroreceptor unloading RSNA, arterial pressure was decreased in a ramp fashion by intravenous administration of sodium nitroprusside until RSNA increased to a maximum (Nishida et al. 2001). The background noise level of RSNA was measured by increasing arterial blood pressure by 50 to $60 \mathrm{~mm} \mathrm{Hg}$ by bolus injection of phenylephrine. The background noise of RSNA was defined as the amplitude during the increase of arterial blood pressure by phenylephrine. The post-mortem signal 
of RSNA was not measured. After the experiment, the transection of left cervical sympathetic trunk was confirmed.

\section{RSNA Analysis}

To quantify the RSNA response, percentage changes in the response were calculated by taking the mean of these values during the pre-transection period of $15 \mathrm{~min}$ as $100 \%$ RSNA.

\section{Statistical Analysis}

RSNA, arterial blood pressure, and heart rate were first averaged within each rat for $1 \mathrm{~min}$; the resulting values were then averaged among rats. One-min averages were compared with the control period, which was an average for $15 \mathrm{~min}$ before transection of the cervical sympathetic trunk. RSNA, arterial blood pressure, and heart rate during left cervical sympathetic trunk transection were compared using repeated-measures analysis of variance. The baroreceptor unloading RSNA before and after transection was compared using paired t-test. Results are presented as means \pm S.E.M.; $P<0.05$ was considered statistically significant.

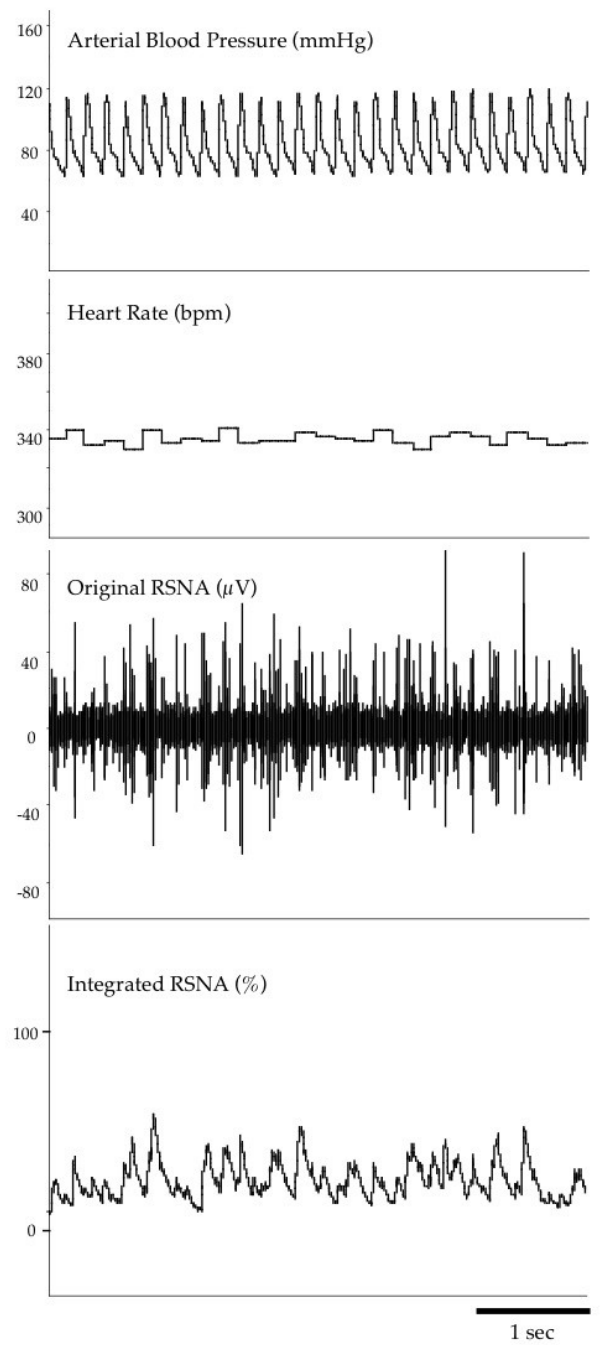

Before Transection

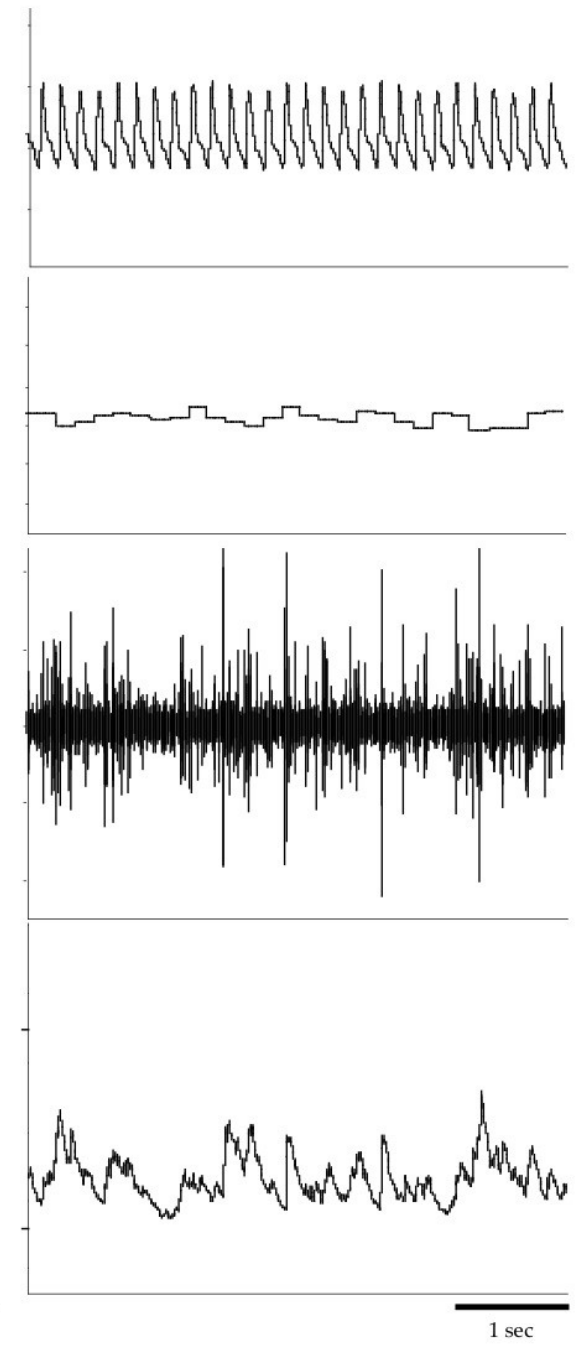

Ten min After Transection

Fig. 1. Representative arterial blood pressure, heart rate, original renal sympathetic nerve activity (RSNA), and integrated RSNA tracings from one rat before and $10 \mathrm{~min}$ after cervical sympathetic trunk transection. Arterial blood pressure, heart rate, and RSNA were virtually identical during left cervical sympathetic trunk transection. 

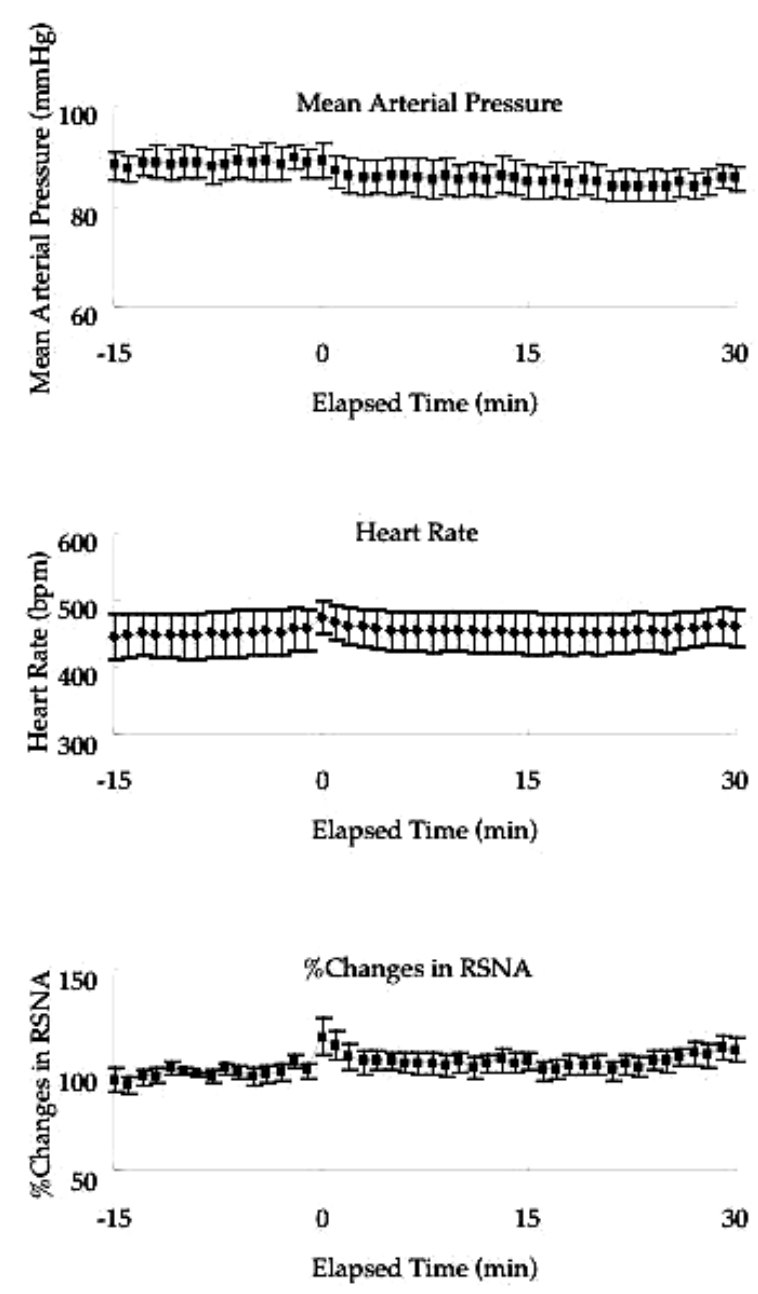

Fig. 2. Average data for mean arterial pressure, heart rate, and renal sympathetic nerve activity (RSNA) in 7 rats. There were no significant changes in mean arterial pressure, heart rate, and RSNA. Elapsed time-zero indicates cervical sympathetic trunk transection. Results are presented as means \pm S.E.M.

\section{Results}

Figure 1 shows representative arterial blood pressure, heart rate, original RSNA and integrated RSNA tracings obtained from one rat before and $10 \mathrm{~min}$ after left cervical sympathetic trunk transection. Arterial blood pressure, heart rate, and RSNA were virtually identical during left cervical sympathetic trunk transection. Figure 2 illustrates average data for mean arterial pressure, heart rate, and RSNA in 7 rats. Although there were transient, but not significant changes in mean arterial pressure, heart rate and RSNA after left cervical sympathetic transection, left cervical sympathetic transection induced no significant changes in mean arterial pressure $(89 \pm 3$ vs. $85 \pm 3 \mathrm{mmHg}$, resting values for $15 \mathrm{~min}$ and values at $10 \mathrm{~min}$ after transection, respectively), heart rate $(452 \pm 33$ vs. $455 \pm 30 \mathrm{bpm}$, resting values for $15 \mathrm{~min}$ and values at 10 min after transection, respectively), and RSNA (100 vs. $105 \pm 5 \%$, resting values for $15 \mathrm{~min}$ and values at 10 min after transection, respectively). The baroreceptor unloading RSNA obtained by decreasing arterial blood pressure by nitroprusside was virtually identical before and after the transection (162 \pm 32 vs. $165 \pm 25 \%$ ).

\section{Discussion}

The major finding of this study is that the left cervical sympathetic trunk transection did not change RSNA in anesthetized rats. Although this observed RSNA might be a result of arterial baroreflex, baroreceptor unloading RSNA obtained by decreasing arterial blood pressure with administration of sodium nitroprusside did not change during the left cervical sympathetic trunk transection indicating that the transection did not change RSNA regardless of arterial baroreflex.

Although we did not measure skin or muscle blood flow in the cervicothoracic region and upper extremity, cervical sympathetic trunk transection increases skin or muscle blood flow in the cervicothoracic region and upper extremity to some extent. This vasodilation might cause the decrease in central venous pressure that induces unloading of cardiopulmonary low-pressure baroreflex and increases RSNA. However, the venous system has the ability to adjust its pressure to the changes in blood volume (Porciuncula et al. 1964). This might be one reason for the unchanged RSNA during cervical sympathetic trunk transection.

Our results suggest that the blockade of cervical sympathetic nerve activity itself may not induce the changes in sympathetic nerve activity in other region. The results indicate that the increase in sympathetic nerve activity in the tibial nerve during SGB might result from infiltration of a local anesthetic to adjacent nerves rather than cervical sympathetic blockade itself. The blockade of vagal impulses from aortic baroreceptors by a local anesthetic during SGB may increase sympathetic nerve activity in other region. Vagal fibers from aortic baroreceptors run immediately adjacent to the stellate ganglion, it is possible that these vagal fibers from aortic baroreceptors may be suppressed by a local anesthetic. Consistent with this possibility, severe hypertension following SGB has been reported in humans (Kimura et al. 2005). In addition, Fagius et al. (1985) demonstrated 
that unilateral lidocaine blockade of glossopharyngeal and vagal fibers from baroreceptors in the neck of humans resulted in increases of muscle sympathetic nerve activity in the peroneal nerve and arterial blood pressure, and that bilateral blockade resulted in marked increases in the muscle sympathetic nerve activity and arterial blood pressure.

Although this study was designed to elucidate a possible mechanism for the increase in muscle sympathetic nerve activity in the tibial nerve following $\mathrm{SGB}$, stellate ganglion is located in T1 level and it is not possible to identify stellate ganglion by a middle incision on the ventral region of the neck. However, it is required to perform thoracotomy and artificial breathing for identifying stellate ganglion. In addition, SGB is mostly performed using the anterior, paratracheal, C-6 level approach in a clinical setting. Cervical sympathetic ganglionectomy causes Horner's syndrome as SGB does (Wax et al. 2004). Thus, we transected cervical sympathetic trunk rather than stellate ganglion.

There are several limitations in this study. One limitation is that we did not measure RSNA during aortic baroreceptor denervation. Further studies are required to elucidate the precise mechanism for the increase in muscle sympathetic nerve activity in the tibial nerve during SGB. Another limitation is that the current data were obtained under urethane anesthesia. Thus, the response of sympathetic nerve activity might be suppressed to some extent. Had RSNA been examined without anesthesia, the result might have been different. Finally, there might be a regional difference between muscle sympathetic nerve activity in the tibial nerve and RSNA. However, this difference might be negligible, since it has been recently reported that muscle sympathetic nerve activity in the tibial nerve parallels RSNA in response to a baroreceptor pressure change (Kamiya et al. 2005).

In conclusion, RSNA was virtually identical during left cervical sympathetic trunk transection. These data suggest that there was no compensatory increase in RSNA when cervical sympathetic trunk was transected and that the increase in sympathetic nerve activity in the tibial nerve during SGB may result from infiltration of a local anesthetic to adjacent nerves rather than SGB itself.

\section{Conflict of Interest}

There is no conflict of interest.

\section{Acknowledgements}

This research was supported in part by The Salt Science Research Foundation, Grant Number 0435 and 0535, and by Kawano Masanori Memorial Foundation for Promotion of Pediatrics, Grant Number 17-4.

\section{References}

FAGIUS J, WALLIN BG, SUNDLOF G, NERHED C, ENGLESSON S: Sympathetic outflow in man after anaesthesia of the glossopharyngeal and vagus nerves. Brain 108: 423-438, 1985.

GUNTAMUKKALA M, HARDY PA: Spread of injectate after stellate ganglion block in man: an anatomical study. Br J Anaesth 66: 643-644, 1991.

HIRAKAWA H, NAKAMURA T, HAYASHIDA Y: Effect of carbon dioxide on autonomic cardiovascular responses to systemic hypoxia in conscious rats. Am J Physiol 273: R747-R754, 1997.

HONMA M, MURAKAMI G, SATO TJ, NAMIKI A: Spread of injectate during C6 stellate ganglion block and fascial arrangement in the prevertebral region: an experimental study using donated cadavers. Reg Anesth Pain Med 25: 573-583, 2000.

IKEDA T, IWASE S, SUGIYAMA Y, MATSUKAWA T, MANO T, DOI M, KIKURA M, IKEDA K: Stellate ganglion block is associated with increased tibial nerve muscle sympathetic activity in humans. Anesthesiology 84: 843-850, 1996.

KAMIYA A, KAWADA T, YAMAMOTO K, MICHIKAMI D, ARIUMI H, MIYAMOTO T, UEMURA K, SUGIMACHI M, SUNAGAWA K: Muscle sympathetic nerve activity averaged over 1 minute parallels renal and cardiac sympathetic nerve activity in response to a forced baroreceptor pressure change. Circulation 112: 384-386, 2005.

KIM JM, ARAKAWA K, VONLINTEL T: Use of the pulse-wave monitor as a measurement of diagnostic sympathetic block and of surgical sympathectomy. Anesth Analg 54: 289-296, 1975. 
KIMURA T, NISHIWAKI K, YOKOTA S, KOMATSU T, SHIMADA Y: Severe hypertension after stellate ganglion block. Br J Anaesth 94: 840-842, 2005.

MALMQVIST EL, BENGTSSON M, SORENSEN J: Efficacy of stellate ganglion block: a clinical study with bupivacaine. Reg Anesth 17: 340-347, 1992.

MILLIGAN NS, NASH TP: Treatment of post-herpetic neuralgia. A review of 77 consecutive cases. Pain 23: 381-386, 1985.

NISHIDA Y, CHEN QH, TANDAI-HIRUMA M, TERADA S, HORIUCHI J: Neuronal nitric oxide strongly suppresses sympathetic outflow in high-salt Dahl rats. J Hypertens 19: 627-634, 2001.

OWEN-FALKENBERG A, OLSEN KS: Continuous stellate ganglion blockade for reflex sympathetic dystrophy. Anesth Analg 75: 1041-1042, 1992.

PORCIUNCULA CI, ARMSTRONG GG JR, GUYTON AC, STONE HL: Delayed compliance in external jugular vein of the dog. Am J Physiol 207: 728-732, 1964.

STEVENS RA, STOTZ A, KAO TC, POWAR M, BURGESS S, KLEINMAN B: The relative increase in skin temperature after stellate ganglion block is predictive of a complete sympathectomy of the hand. Reg Anesth Pain Med 23: 266-270, 1998.

WAX MK, SHILEY SG, ROBINSON JL, WEISSMAN JL: Cervical sympathetic chain schwannoma. Laryngoscope 114: 2210-2213, 2004. 